\title{
Primary posterior continuous curvilinear capsulorhexis combined with diffractive multifocal intraocular lens implantation
}

M Ouchi

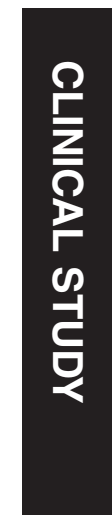

\begin{abstract}
Purpose To evaluate the effect of mild posterior capsule opacity (PCO) on visual acuity (VA) in eyes implanted with a diffractive multifocal intraocular lens (IOL) compared with a monofocal IOL, and the effect of posterior continuous curvilinear capsulorhexis (PCCC) combined with diffractive multifocal IOL implantation.
\end{abstract}

Methods For the initial evaluation, we compared charge-coupled device (CCD) camera photographs taken through both a monofocal IOL-loaded model eye and a diffractive IOL-loaded model eye under the conditions of both with and without an opaque filter for the simulation of mild PCO. The clinical evaluation involved 20 patients who underwent bilateral implantation of the same diffractive multifocal IOL. In all 20 cases, PCCC was performed in 1 eye (PCCC group) and not performed in the fellow eye (NCCC group). Postoperative clinical results were then compared between the two groups.

Results The CCD photographs revealed that the diffractive IOL-loaded eye was more strongly affected by the simulated PCO than was the monofocal IOL-loaded eye. In the clinical setting, the PCCC group registered better results than NCCC group in distance and near VA, low-contrast VA, and contrast sensitivity testing.

Conclusions Diffractive multifocal IOLs tend to be more influenced by mild PCO than do monofocal IOLs, and PCCC prior to IOL implantation can contribute to the avoidance of this effect.

Eye (2016) 30, 95-101; doi:10.1038/eye.2015.201; published online 23 October 2015

\section{Introduction}

Since 2006, it has been reported that when using a diffractive multifocal intraocular lens (diffractive IOL), 'hazy vision' or 'waxy vision' can sometimes occur and become a problem due to the associated decrease of optical contrast that subsequently arises. ${ }^{1-3}$ It is also well known that even small ametropia, dry eye, or mild posterior capsule opacity $(\mathrm{PCO})$ may have a role in these visual symptoms. ${ }^{4,5}$ Therefore, strict management of these problems are recommended for eyes implanted with a diffractive IOL. However, most patients who experience diffractive IOL-associated hazy vision or waxy vision complain of these symptoms in the immediate postoperative period, possibly due to posterior capsule folds or the existence of residual epithelial cells as opposed to the proliferation of crystalline epithelial cells, as that proliferation does not occur until long after surgery. Thus, the residual epithelial cells or posterior capsule folds, as opposed to a postoperative proliferation of crystalline epithelial cells immediately after cataract surgery may have a role for cases in which PCO is theorized to be a trigger for those symptoms. However, in many cases the underlying cause of the postoperative hazy vision cannot be identified. And even in cases in which mild PCO is suspected to be a primary cause of the hazy vision, surgeons cannot be certain as to whether the yttrium aluminum garnet (YAG) laser posterior capsulotomy procedure will effectively decrease that haze until after the YAG procedure. Moreover, even though some cases ultimately require a replacement of diffractive IOLs with monofocal IOLs, it is very difficult to surgically exchange the IOLs after YAG capsulotomy.
Ouchi Eye Clinic, Kyoto, Japan

Correspondence:

M Ouchi, Ouchi Eye Clinic, 47-1 Karahashi,

Rajomon-cho, Minami-ku, Kyoto 601-8453, Japan

Tel: +81 756627117 ;

Fax: +8175662 7118 E-mail: mouchi@skyblue. ocn.ne.jp

Received: 22 February 2015 Accepted in revised form: 16 August 2015 Published online: 23 October 2015 
In this study, we first evaluated the influence of mild opacity existing on the optical axis towards optical quality in a diffractive IOL implanted eye compared with a monofocal IOL implanted eye using an optical simulation in the laboratory setting. Next, we evaluated the clinical results of primary posterior continuous curvilinear capsulorhexis (PCCC) for the purpose of avoiding posterior capsule folds and cleaning up residual lens epithelial cells to avoid a decrease of contrast associated with the diffractive multifocal IOL implantation.

\section{Materials and methods}

\section{Laboratory settings}

An in-house produced model eye ${ }^{6}$ was prepared, and a charge-coupled device (CCD) camera (ARTCAM-150P; ARTRAY, Tokyo, Japan) was mounted to the eye. Test charts for visual acuity (VA) were set up $4 \mathrm{~m}$ in front of the model eye for distance VA, and at $50-\mathrm{cm}$ and $40-\mathrm{cm}$ in front of the model eye for near VA, and photographs were then taken through the model eye after it was alternately loaded with an AcrySof IQ monofocal IOL (SN60WF; Alcon Laboratories, Inc., Fort Worth, TX, USA; monofocal IOL eye) and a ReSTOR+3D diffractive IOL (SN6AD1; Alcon Laboratories; diffractive IOL eye) and under the following two conditions: (1) photos taken with no filter and (2) photos taken with an opaque filter (Foggy B filter; Kenko Tokina, Tokyo, Japan) for the simulation of mild PCO.

\section{Clinical setting}

The clinical evaluation involved 40 eyes of 20 patients (9 males and 11 females; age range: $41-75$ years, mean age: 63.5 years) who underwent bilateral transplantation of the ReSTOR+3D IOL. Exclusion criteria included intraoperative complication and/or eyes with astigmatism $>1.5$ diopters (D), and patient screening was conducted until 20 cases were enrolled into the study. All cases underwent PCCC prior to IOL implantation in 1 eye (PCCC group), yet no PCCC prior to IOL implantation in the fellow eye (NCCC group), and the postoperative clinical results were then compared between the two groups. All procedures were approved by the Ethics Committee of Ouchi Eye Clinic, and informed consent was obtained from all subjects. This study was conducted in accordance with the tenets set forth in the Declaration of Helsinki.

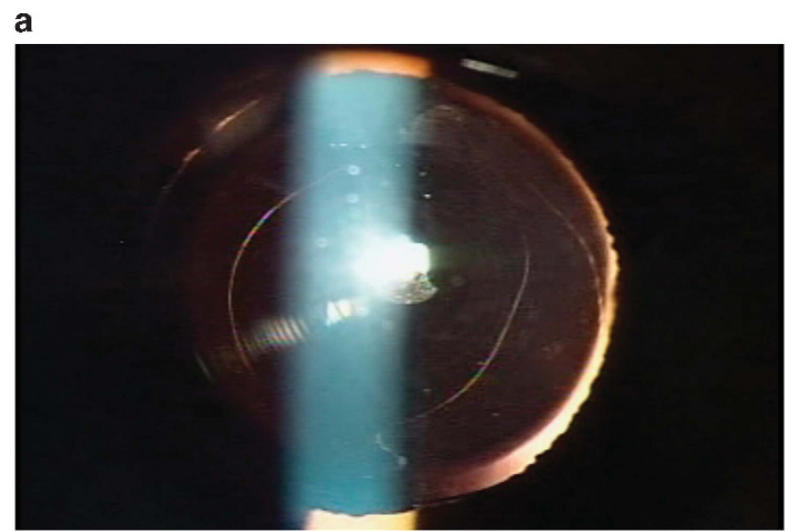

b

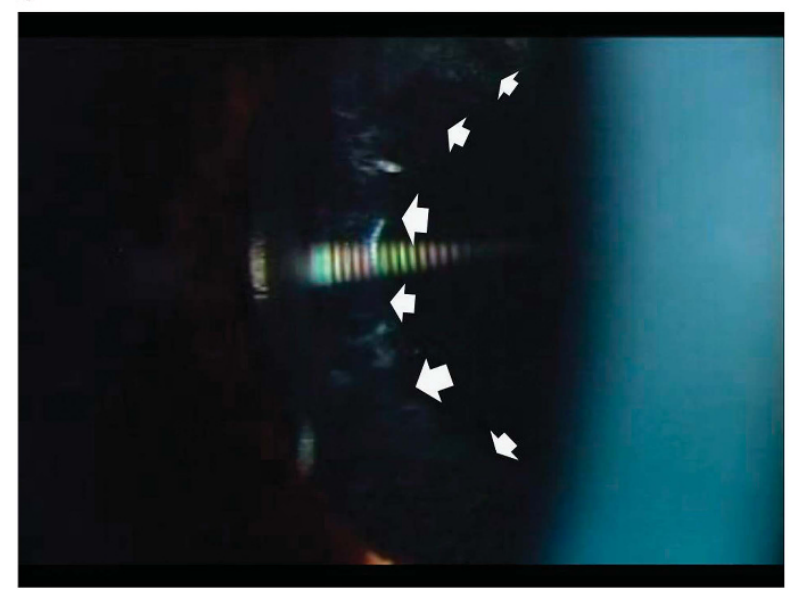

Figure 1 Slit-lamp examination of a PCCC eye (right eye) at 1-day postoperative. (a) PCCC can be easily observed using retroillumination. (b) Macro photograph of the same eye illustrating the difference of transparency between the PCCC area and the remaining posterior capsule bordering the edge of the PCCC (arrowhead). PCCC, posterior continuous curvilinear capsulorhexis.

\section{Randomization}

In all cases, the second operation was performed at 1 week after the first operation. Each eye was randomly assigned to either the PCCC group or the NCCC group via the envelope selection method. The data analyzer randomly picked and opened one of two envelopes (P- or N-lettered card inside) at the patient's last visit prior to the first eye operation, and both the surgeon and patients were aware of which eye was to undergo PCCC immediately after assignment. Therefore, this study was not a blind study.

\section{Surgical technique}

A 2.2-mm, clear corneal incision was made by use of a 2.2-mm disposable steel knife (Slit knife 2.2DB; Alcon Laboratories). Phacoemulsification was performed by use of the Infiniti Vision System (Alcon Laboratories) with an 
attached 0.9-mm mini flare ABS phaco tip, $45^{\circ}$ KELMAN, and ultra-sleeve (Alcon Laboratories). After removal of the cortex, the following procedures were conducted depending on the groups. In the PCCC group, after a viscoelastic agent was injected into the capsule bag, a posterior capsule tear was made using a 25-gauge needle and viscoelastic agent was injected between the posterior capsule and anterior vitreous membrane, followed by posterior capsulorhexis using capsular forceps. The IOL was then inserted into the capsular bag. In the NCCC group, the IOL was inserted immediately after injection of viscoelastic agent into the capsular bag post cortex removal. Slit-lamp examination of a PCCC eye at 1-day postoperative is shown in Figure 1.

\section{Outcome measurement}

In all eyes, uncorrected distance VA (UDVA), bestcorrected distance VA (CDVA), uncorrected near VA (UNVA), best-corrected near VA (CNVA), respectively, low-contrast VA testing with 12.5 and $6 \%$ contrast, and contrast sensitivity tests were carried out at 1-day, 1-month, and 6-months postoperative. Optical coherence tomography (OCT) was also performed at 1-day and 6-months postoperative. Distance VA and low-contrast VA was measured with a VA system chart (SC-1600; NIDEK, Gamagori, Japan), and contrast sensitivity testing was conducted with a CSV-1000 (VectorVision, Greenville, $\mathrm{OH}, \mathrm{USA}$ ) test instrument under a mesopic non-glare condition. All patients completed a questionnaire pertaining to their overall opinion of the quality of distance and near vision (response scale: $1=$ very poor, 2 = poor, $3=$ acceptable, $4=$ good, and $5=$ very good) at the 1 -month postoperative visit.

The Mann-Whitney $U$-test was used to compare the preoperative baseline data and postoperative corneal astigmatism and spherical equivalent. The Wilcoxon signed-ranks test was used to compare the measurements of all the remaining postoperative data. The results are presented as mean \pm SEM unless otherwise noted. Any differences with a $P$-value of $<0.05$ were considered to be statistically significant.

\section{Results}

\section{Laboratory settings}

In the condition without the opaque filter, test charts for distance VA could be distinguished up to the five large Landolt rings, both in the monofocal IOL eye and the diffractive IOL eye (Figure $2 a$ and $b$ ). In the diffractive IOL eye, photographs indicated clearer Landolt rings in the 50-cm and 40-cm test charts for near VA than in the monofocal IOL eye (Figure 2a and b). In the condition with the opaque filter, photographs both through the monofocal IOL eye and the diffractive IOL eye showed lower contrast compared with the nonfilter photos. However, decrease of contrast was greater in the diffractive IOL eye, with only three distinguishable large Landolt rings, while still five large Landolt rings could be distinguished in the monofocal IOL eye (Figure $2 \mathrm{c}$ and $\mathrm{d}$ ).

\section{Clinical setting}

All enrolled eyes underwent the scheduled examinations. No significant difference was observed between the PCCC group and the NCCC group in preoperative baseline data including UDVA $(0.49 \pm 0.34$ and $0.48 \pm 0.41$, respectively), CDVA $(0.08 \pm 0.16$ and $0.18 \pm 0.22$, respectively), keratometric astigmatism $(0.85 \pm 0.39 \mathrm{D}$ and $0.75 \pm 0.35 \mathrm{D}$, respectively), spherical equivalent $(-0.43 \pm 3.16 \mathrm{D}$ and $-0.56 \pm 4.21 \mathrm{D}$, respectively), and pupil size $(3.76 \pm 0.82$ and $3.61 \pm 0.81$, respectively). In addition, no significant difference was observed in regard to postoperative corneal astigmatism $(0.68 \pm 0.40 \mathrm{D}$ and $0.55 \pm 0.36 \mathrm{D}$, respectively), spherical equivalent $(-0.43 \pm 3.16 \mathrm{D}$ and $-0.56 \pm 4.21 \mathrm{D}$, respectively), and pupil size $(3.70 \pm 0.82 \mathrm{~mm}$ and $3.61 \pm 0.81 \mathrm{~mm}$, respectively) between the two groups $(P=0.34,0.36$, and 0.35 respectively). No intraoperative or postoperative complication related to PCCC (ie, cystoid macular edema or retinal tears) was observed. YAG posterior capsulotomy was performed in one eye in the NCCC group owing to a strong complaint during the observation period.

Postoperative UDVA were $-0.07 \pm 0.11,-0.05 \pm 0.18$, and $-0.06 \pm 0.11$, respectively, in the PCCC group, and $0.005 \pm 0.18,-0.01 \pm 0.18$, and $-0.003 \pm 0.10$ in NCCC group, respectively, and PCCC group registered significant better at 1-day postoperative (Figure 3a). The CDVA findings were significantly better in PCCC group than in the NCCC group at 1-day and 6-month postoperative $(-0.17 \pm 0.06,-0.12 \pm 0.08$, and $-0.15 \pm 0.07$, respectively in the PCCC group, and $-0.05 \pm 0.11,-0.07 \pm 0.1$, and $-0.07 \pm 0.1$, respectively, in the NCCC group; Figure 3b). The PCCC group registered significantly better mean UNVA than did the NCCC group at all postoperative period $(0.097 \pm 0.16$, $0.098 \pm 0.09$, and $0.12 \pm 0.17$, respectively, in the PCCC group and $0.18 \pm 0.17,0.18 \pm 0.19$, and $0.22 \pm 0.14$, respectively, in the NCCC group; Figure 3c). The mean CNVA findings were also better in PCCC group, with significance observed at 1-day and 6-month postoperative $(-0.11 \pm 0.21,-0.07 \pm 0.09$, and $-0.08 \pm 0.09$, respectively, in the PCCC group and $-0.01 \pm 0.17,-0.02 \pm 0.08$, and $0 \pm 0.08$, respectively, in the NCCC group; Figure $3 \mathrm{~d}$ ). In low-contrast $\mathrm{VA}$, both 12.5 and $6 \%$ contrast VA was found to be better in the PCCC group than in the 
a

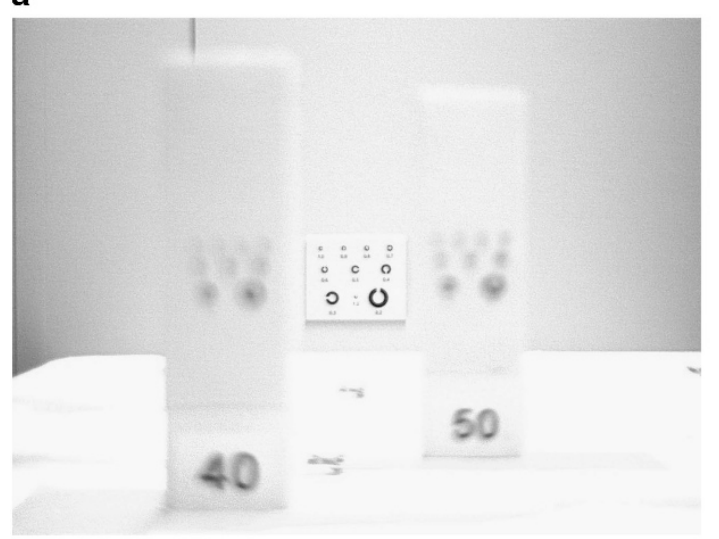

C

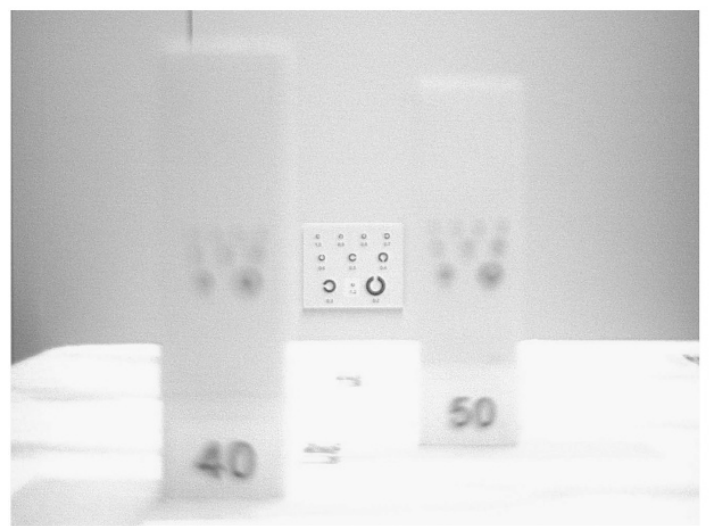

b

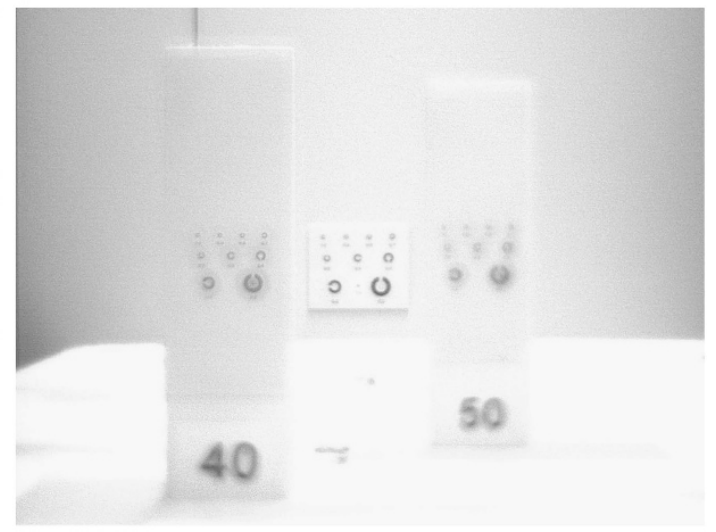

d

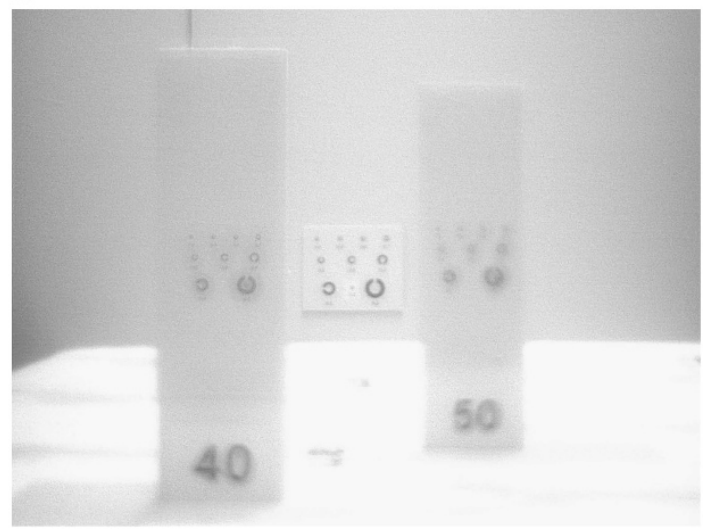

Figure 2 Comparison of images between through monofocal IOL and diffractive IOL loaded model eyes both with and without simulated mild PCO using an opaque filter. (a) Photograph taken through a monofocal IOL without simulated PCO. (b) Photograph taken through a diffractive IOL without simulated PCO. (c) Photograph taken through a monofocal IOL with simulated PCO. (d) Photograph taken through a diffractive IOL with simulated PCO. Without the opaque filter, the Landolt rings of the distance test chart (center) could be distinguished up to the five large rings, both in the monofocal IOL (a) and diffractive IOL (b) loaded model eye. With the opaque filter, contrast decreased in both of the IOL loaded model eyes. However, up to five large rings in the distance VA test chart could still be distinguished through the monofocal IOL loaded eye (c) whereas only three large rings could be distinguished and with strong degradation of image quality through the diffractive IOL loaded eye (d). Moreover, the $40 \mathrm{~cm}$ (left) and $50 \mathrm{~cm}$ (right) near VA test chart showed further image degradation through the diffractive IOL loaded eye (d). VA, visual acuity; diffractive, diffractive multifocal; IOL, intraocular lens; PCO, posterior capsule opacity.

NCCC group. There were significant differences at all postoperative periods in $12.5 \%$ low-contrast VA, and at 1-day and 6-month postoperative in the $6 \%$ low-contrast VA (Figure 4a and b).

The PCCC group registered better contrast sensitivity, with significant difference in 12 and 18 cycles per degree (cpd) at 1-day postoperative, and $12 \mathrm{cpd}$ at 6 -months postoperative (Figure 5).

The results of the patient questionnaire revealed that although all 20 patients $(100 \%)$ rated their quality of vision as 4 or higher (good or very good) among the 5 items for their PCCC eye, 4 patients $(20 \%)$ rated their quality of vision as 3 (acceptable) and 2 patients (10\%) rated their quality of vision as 2 (poor) for their NCCC eye.

\section{Discussion}

In our laboratory setting, the diffractive IOL was more strongly affected by mild PCO than was the monofocal IOL in terms of visual quality. Moreover, better postoperative clinical results were observed and higher patient satisfaction was obtained when primary PCCC was performed at the time of surgery for removal of crystalline epithelial cells.

In many countries, the diffractive IOL is chosen for implantation due to the fact that it offers higher near addition and good reading VA, however, the hazy or waxy vision caused by the decrease of contrast sometimes becomes a problem. ${ }^{1-3}$ Moreover, mild ametropia, dry eye, and mild PCO are reported as major triggers for these visual symptoms; Woodward et $a l^{4}$ reported that PCO 

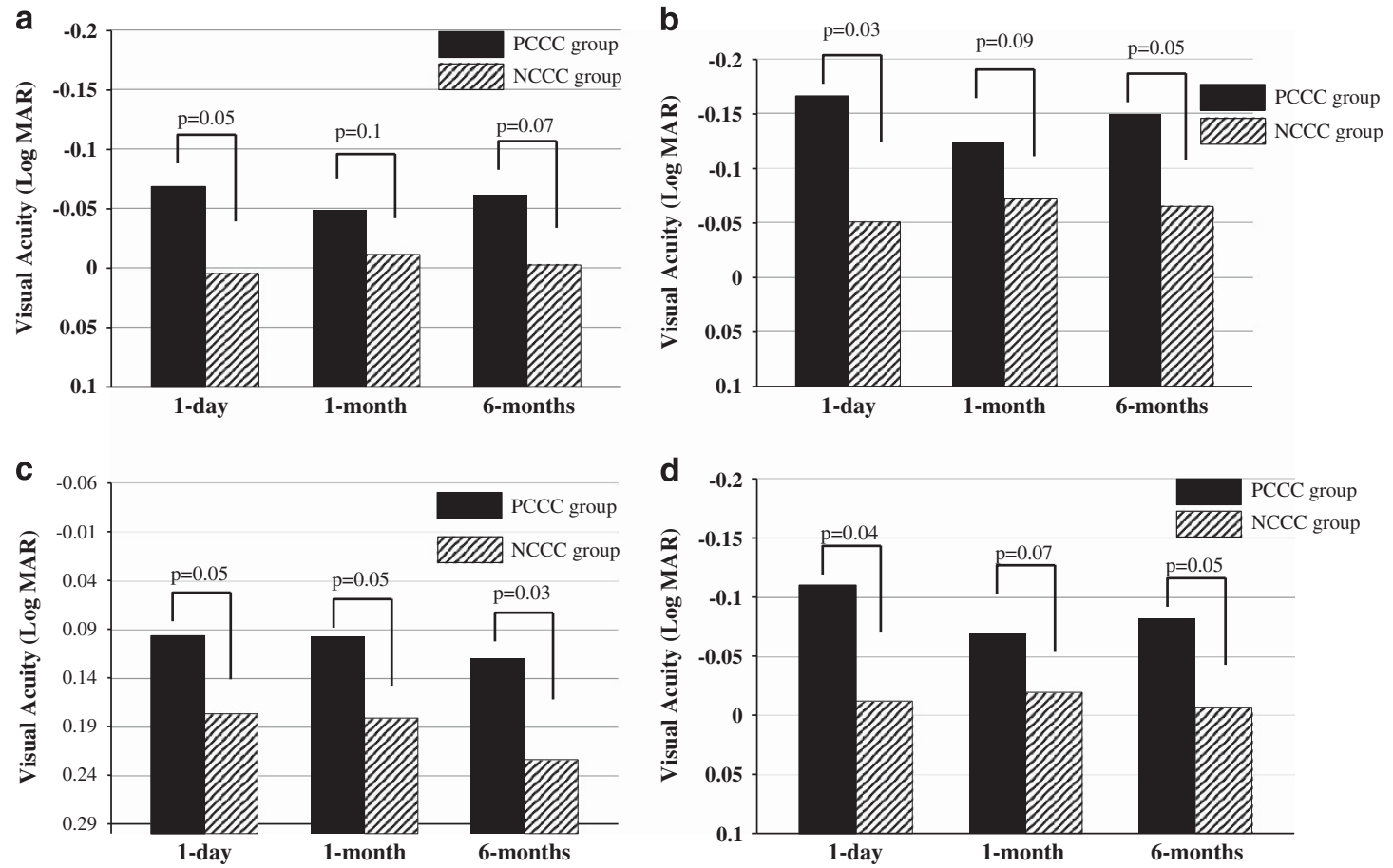

Figure 3 Uncorrected (a) and best-corrected (b) postoperative distance VA. Uncorrected (c) and best-corrected (d) postoperative near VA.

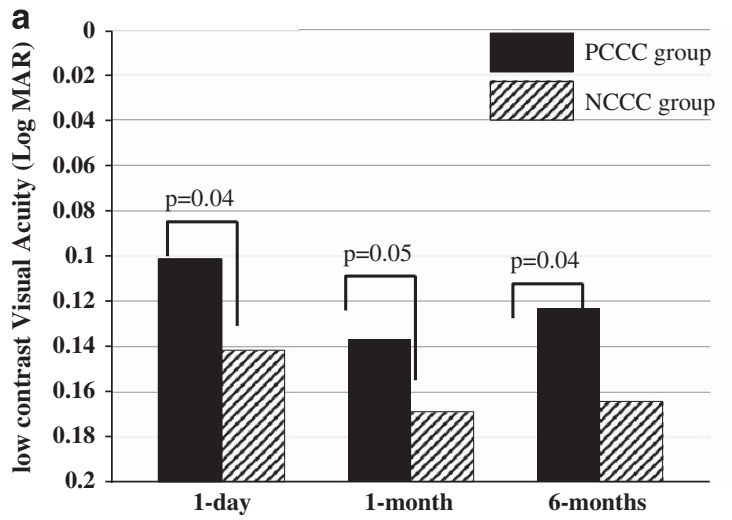

b

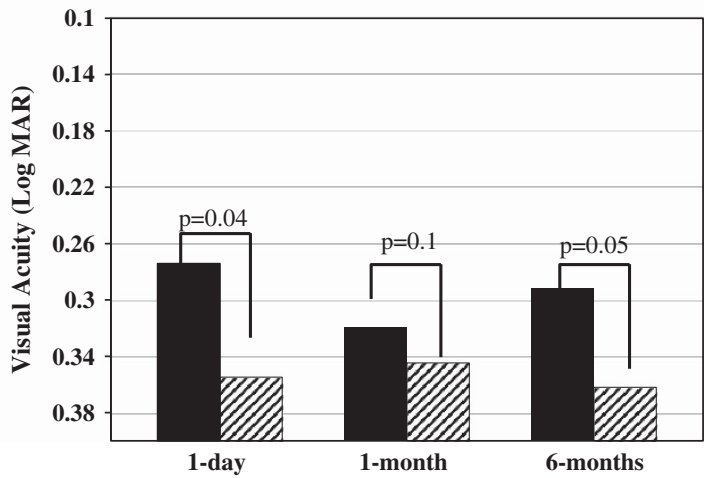

Figure 4 Postoperative low-contrast VA. (a): 12.5\% low-contrast VA, (b): 6\% low-contrast VA.

was observed in 54\% of their hazy vision cases and deVries et $a l^{5}$ reported PCO in $67 \%$ of their hazy vision cases. However, patients usually complain of hazy vision from the very early postoperative period, so it is difficult to explain the underlying mechanism of the hazy vision with proliferation of crystalline lens epithelial cells. It is possible that a small number of remaining cells or posterior capsule folds become critical for visual quality from immediately after surgery in diffractive IOL implanted eyes.

In this study, we first investigated in the laboratory setting whether or not a diffractive IOL is more strongly affected by mild opacity on visual axis than is a monofocal IOL. Elgohary and Beckingsale ${ }^{7}$ reported that patients who undergo multifocal IOL implantation tend to be less tolerant of PCO-induced loss of visual function and tend to undergo YAG laser therapy for the treatment of early-stage PCO. Thus, even mild PCO, which seldom affects a monofocal IOL implanted eye, might lead to hazy vision in a diffractive IOL implanted eye. However, that theory has never been verified until now, even though it has been previously speculated. ${ }^{8}$ The results of our laboratory study ultimately verified that speculation.

Among the above-described triggers of hazy vision, a therapeutic effect can be simulated in regard to ametropia and dry eye using various temporal methods. However, 

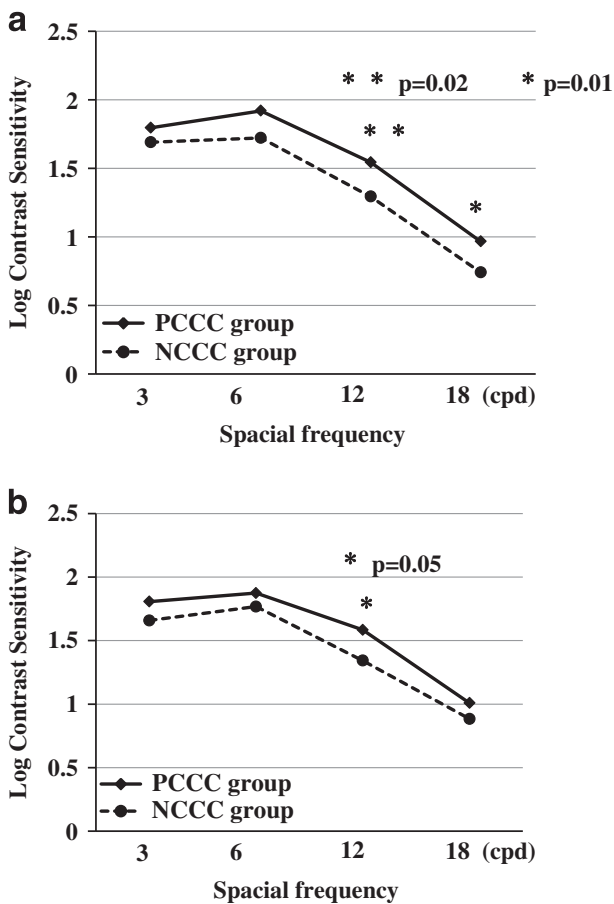

Figure 5 Contrast sensitivity at (a) 1-day postoperative and (b) 6-months postoperative.

the effect of the removal of PCO cannot be simulated in order for a surgeon to know whether or not mild PCO contributes to the hazy vision, and the efficacy of YAG laser treatment can only be known postoperatively. However, secondary IOL implantation into the capsule bag is very troublesome in cases following YAG posterior capsulotomy, though some cases do require the replacement of a diffractive IOL with a monofocal IOL. On this matter, opinions have been divided, as some reports state that YAG treatment should be done sooner because of neural adaptation, ${ }^{7,8}$ whereas on the other hand, Woodward et al ${ }^{4}$ reported that surgeons should hold off on performing the YAG treatment until they are assured that the patient does not require an IOL

exchange. Moreover, Vrijman $e t \mathrm{al}^{9}$ reported that $>0.5 \mathrm{D}$ refractive shift was seen in $7 \%$ of diffractive IOL implantation cases that underwent YAG treatment, so it appears that this dilemma has yet to be resolved.

To address these problems, we next evaluated the effect of primary PCCC for diffractive IOL implantation in the clinical setting. Primary PCCC was first reported by Castaneda et $\mathrm{al}^{10}$ in 1992, and has now become a primary procedure in pediatric cataract surgery. ${ }^{11-13}$ Moreover, surgeons are increasingly introducing this procedure in cataract surgery for adult patients, and many reports have been published with good clinical results since $2003 .{ }^{14-18}$

In this study, we applied the PCCC technique for diffractive IOL Implantation. Performing primary PCCC eliminates the need to wonder if YAG laser treatment should be performed in cases of postoperative hazy vision, thus decreasing the surgeon's stress in the postoperative follow-up. Moreover, this clinical study revealed that both distance and near VA registered significantly better in the PCCC group than in the NCCC, and that low-contrast VA and contrast sensitivity also registered better in the PCCC group. As can be seen in Figure $2 b$, PCO that might be remaining lens epithelial cells exists even at the very early postoperative period. Therefore, avoiding capsule folds or primary removal of lens epithelial cells on the visual axis might have provided an advantage in the PCCC group. Primary PCCC for monofocal IOL implantation has previously been reported and described for smaller optical axis opacity or low PCO rate, ${ }^{14-18}$ thus prompting the possibility that the potential optical loss associated with a diffractive IOL implanted eye would benefit strongly from PCCC.

In this study, no PCCC-related intraoperative or postoperative complications, such as cystoid macular edema or vitreous loss, were observed. Moreover, it was previously reported that PCCC had no effect on the blood-aqueous barrier, ${ }^{19}$ the blood-retinal barrier, ${ }^{20}$ or postoperative macular thickness. ${ }^{21}$ On the other hand, it is known that proliferation of lens epithelial cells can occur on the posterior surface of an IOL, and even after PCCC in some cases. ${ }^{17}$ Moreover, they can also proliferate with a foothold on the anterior vitreous membrane in young cases without anterior vitreous detachment. In this case series, even though no measures were taken to meet these situations, some cases revealed opacity on the posterior surface of the IOL. To address this matter, Menapace ${ }^{17,22}$ reported the combined surgery of PCCC and posterior optic capture, and anterior vitrectomy has also been simultaneously used with PCCC in many pediatric cataract cases in which the strong proliferation of lens epithelial cells tends to occur. ${ }^{13}$ These additional procedures may provide a clue to advance the long-term results of primary PCCC combined diffractive IOL implantation.

It is important to note that this present study did have some limitations, as it was not performed as a blind study. Each patient was well aware as to which eye had undergone PCCC, and there is a possibility this knowledge did affect the answers in patient questionnaire. However, the protocol that was used is imperative for this kind of clinical study.

In conclusion, the implanted diffractive IOL was found to come under the influence of mild PCO compared with the implanted monofocal IOL. Primary PCCC to encourage transparency of the optical axis was found to be effective for postoperative visual function and patient satisfaction. 


\section{Summary}

\section{What was known before}

- Contrast sensitivity in eyes with diffractive multifocal IOL is lower than with mono focal IOL.

- Multi focal IOL implanted patients tend to complain visual symptom with early PCO and undergo YAG capsulotomy for it.

\section{What this study adds}

- Images behind the diffractive IOL are susceptible to mild opacity on optic axis.

- Primary PCCC is effective for increase early postoperative results and patient satisfaction in diffractive IOL implanted eye.

\section{Conflict of interest}

The author declares no conflict of interest.

\section{Acknowledgements}

I thank John Bush for reviewing the manuscript.

\section{References}

1 Souza CE, Gerente VM, Chalita MR, Soriano ES, Freitas LL, Belfort R Jr. Visual acuity, contrast sensitivity, reading speed, and wavefront analysis: pseudophakic eye with multifocal IOL (ReSTOR) versus fellow phakic eye in non-presbyopic patients. J Refract Surg 2006; 22: 303-305.

2 Vingolo EM, Grenga P, Iacobelli L, Grenga R. Visual acuity and contrast sensitivity: AcrySof ReSTOR apodized diffractive versus AcrySof SA60AT monofocal intraocular lenses. J Cataract Refract Surg 2007; 33: 1244-1247.

3 Blaylock JF, Si Z, Aitchison S, Prescott C. Visual function and change in quality of life after bilateral refractive lens exchange with the ReSTOR multifocal intraocular lens. J Refract Surg 2008; 24: 265-273.

4 Woodward MA, Randleman JB, Stulting RD. Dissatisfaction after multifocal intraocular lenses implantation. J Cataract Refract Surg 2009; 35: 992-997.

5 de Vries NE, Webers CA, Touwslager WR, Bauer NJ, de Brabander J, Berendschot TT, Nuijts RM. Dissatisfaction after implantation of multifocal intraocular lenses. J Cataract Refract Surg 2011; 37: 859-865.

6 Yaguchi S, Souda M. Visual simulation of retinal images through new generation multifocal intraocular lenses. Jpn J Cataract Refract Surg 2009; 23: 214-223.

7 Elgohary MA, Beckingsale AB. Effect of posterior capsular opacification on visual function in patients with monofocal and multifocal intraocular lenses. Eye 2008; 22: 613-619.

8 Shah VC, Russo C, Cannon R, Davidson R, Taravella MJ. Incidence of Nd:YAG capsulotomy after implantation of AcrySof multifocal and monofocalintraocular lenses: a case controlled study. J Refract Surg 2010; 26: 565-568.
9 Vrijman V, van der Linden JW, Nieuwendaal CP, van der Meulen IJ, Mourits MP, Lapid-Gortzak R. Effect of Nd: YAG laser capsulotomy on refraction in multifocal apodized diffractive pseudophakia. J Refract Surg 2012; 28: 545-550.

10 Castaneda VE, Legler UF, Tsai JC, Hoggatt JP, Assia EI, Hogan C, Apple DJ. Posterior continuous curvilinear capsulorhexis; an experimental study with clinical applications. Ophthalmology 1992; 99: 45-50.

11 Gimbel HV, DeBroff BM. Posterior capsulorhexis with optic capture: maintaining a clear visual axis after pediatric cataract surgery. J Cataract Refract Surg 1994; 20: 658-664.

12 Koch DD, Kohnen T. Retrospective comparison of techniques to prevent secondary cataract formation after posterior chamber intraocular lens implantation in infants and children. J Cataract Refract Surg 1997; 23: 657-663.

13 Ellis FJ. Management of pediatric cataract and lens opacities. Curr Opin Ophthalmol 2002; 13: 33-37.

14 Georgopulos M, Menapace R, Findl O, Petternel V, Kiss B, Rainer G. After-cataract in adults with primary posterior capsulorhexis; comparison of hydrogel and silicone intraocular lenses with round edges after 2 years. J Cataract Refract Surg 2003; 29: 955-960.

15 Stifter E, Menapace R, Luksch A, Neumayer T, Sacu S. Anterior chamber depth and change in axial intraocular lens position after cataract surgery with primary posterior capsulorhexis and posterior optic buttonholing. J Cataract Refract Surg 2008; 34: 749-754.

16 Vock L, Menapace J, Stifter E, Bühl W, Georgopoulos M. Effect of primary posterior continuous curvilinear capsulorhexis on clinical performance of ACR6D SE single-piece hydrophilic acrylic intraocular lenses. J Cataract Refract Surg 2007; 33: 628-634.

17 Menapace R. Routine posterior optic buttonholing for eradication of posterior capsule opacification in adults; report of 500 consecutive cases. J Cataract Refract Surg 2006; 32: 929-943.

18 Stifter E, Menapace R, Kriechbaum K, Vock L, Luksch A. Effect of primary posterior continuous curvilinear capsulorhexis with and without posterior optic buttonholing on postoperative anterior chamber flare. J Cataract Refract Surg 2009; 35: 480-484.

19 Stifter E, Menapace R, Luksch A, Neumayer T, Vock L, Sacu S. Objective assessment of intraocular flare after cataract surgery with combined primary posterior capsulorhexis and posterior optic buttonholing in adults. Br J Ophthalmol 2007; 91: 1481-1484.

20 De Groot V, Hubert M, Van Best JA, Engelen S, Aelst VS, Tassignon JM. Lack of fluorophotometric evidence of aqueous-vitreous barrier disruption after posterior capsulorhexis. J Cataract Refract Surg 2003; 29: 2330-2338.

21 Stifter E, Menapace R, Neumayer T, Luksch A. Macular morphology after cataract surgery with primary posterior capsulorhexis and posterior optic buttonholing. Am J Ophthalmol 2008; 146: 15-22.

22 Menapace R. Posterior capsulorhexis combined with optic buttonholing: an alternative to standard in-the-bag implantation of sharp-edged intraocular lenses? A critical analysis of 1000 consecutive cases. Graefes Arch Clin Exp Ophthalmol 2008; 246: 787-801. 